\title{
The effectiveness of prophylactic ipsilateral central neck dissection in selected patients who underwent total thyroidectomy for clinically node-negative unilateral papillary thyroid carcinoma
}

\author{
Jin Gu Kang ${ }^{1}$, Young Ah Kim ${ }^{1}$, Jung Eun Choi ${ }^{2}$, Soo Jung Lee ${ }^{2}$, Su Hwan Kang ${ }^{2}$ \\ ${ }^{1}$ Department of Surgery, Yeungnam University Hospital, Daegu, Korea \\ ${ }^{2}$ Department of Surgery, Yeungnam University College of Medicine, Daegu, Korea
}

Received: January 23, 2020

Revised: March 17, 2020

Accepted: March 29, 2020

Corresponding author:

Su Hwan Kang

Department of Surgery, Yeungnam

University College of Medicine, 170

Hyunchoong-ro, Namgu, Daegu

42415, Korea

Tel: +82-53-620-3287

Fax: +82-53-624-1213

E-mail: kangsuhwan@yu.ac.kr
Background: Prophylactic central neck dissection (CND) in clinically node-negative (CNO) papillary thyroid carcinoma (PTC) remains controversial. The purpose of this study was to evaluate the benefits of prophylactic ipsilateral CND compared with bilateral CND in total thyroidectomy for cNO unilateral PTC.

Methods: We retrospectively enrolled 174 patients who underwent total thyroidectomies with prophylactic CND for cNO unilateral PTC between January 2009 and May 2010. The prophylactic CND patients were divided into group 1, the ipsilateral CND group $(n=74)$, and group 2, the bilateral CND group $(n=100)$. The incidence of central lymph node metastasis (CLNM) and postoperative complications, such as hypoparathyroidism, recurrent laryngeal nerve injury, and recurrence were assessed.

Results: CLNM was found in $22(29.8 \%)$ in group 1 and 69 (69\%) in group 2. The incidence of postoperative severe hypocalcemia less than 7.0 was also significantly different (six patients [8.1\%] in group 1 and 23 [23\%] in group 2; $p=0.009$ ). Permanent hypoparathyroidism was significantly more frequent in group 2 ( $4.1 \%$ vs. 19\%; $p=0.005$ ). However, the incidence of transient hypoparathyroidism, recurrence, and recurrent laryngeal nerve injury was not significantly different. Conclusion: Prophylactic ipsilateral CND has advantage not only to reduce incidence of some complications but also to have similar recurrence rate compared with bilateral CND. We suggest that prophylactic ipsilateral CND may be safe and effective for selected patients undergoing total thyroidectomy for cNO unilateral PTC.

Keywords: Hypoparathyroidism; Lymph node; Neck dissection; Papillary thyroid carcinoma

\section{Introduction}

The treatment of differentiated thyroid carcinoma has shown excellent outcomes, with a $0.6 \%$ recurrence rate and over $95 \% 10$ year disease-free survival [1]. Nevertheless, there are complications associated with total thyroidectomies, such as hypoparathy- roidism, recurrent laryngeal nerve and external branch of the superior laryngeal nerve injury, and locoregional recurrence. The risk of these complications may increase with the extent of surgery. Recently, there has been a growing interest in functional complications after total thyroidectomy. The typical long-term functional complication after total thyroidectomy is permanent

Copyright (C) 2020 Yeungnam University College of Medicine

This is an Open Access article distributed under the terms of the Creative Commons Attribution Non-Commercial License (http://creativecommons.org/licenses/by-nc/4.0/) which permits unrestricted non-commercial use, distribution, and reproduction in any medium, provided the original work is properly cited. 
hypoparathyroidism, with a 10\%-60\% incidence [2]. Most endocrine surgeons follow a parathyroid preservation principle during thyroidectomy; however, hypoparathyroidism can occur because of devascularization, thermal or mechanical damage, and inadvertent removal of the parathyroid gland. Permanent hypoparathyroidism can lead to symptomatic hypocalcemia, the need for lifetime calcium supplementation, and seriously impact the patient's quality of life. Furthermore, the incidence of permanent hypoparathyroidism after total thyroidectomy is greatly influenced by the experience of the operator and the decision of whether or not to perform central neck dissection (CND).

Central lymph node metastases (CLNM) have been reported in approximately $30 \%-80 \%$ of the patients with papillary thyroid carcinoma (PTC) [3]. It is difficult to detect metastatic central lymph node ( $\mathrm{LN}$ ) by ultrasound preoperatively because, while the specificity is $80 \%-93 \%$, the sensitivity is only $53 \%-61 \%$ [ 4 ]. Tumor size and extrathyroidal extension are known risk factors for ipsilateral CLNM. And ipsilateral CLNM is a significant factor in contralateral LNM [3,5-8]. Prophylactic CND is defined as the removal of level VI LNs, which includes the pretracheal, prelaryngeal, and paratracheal LNs. Prophylactic CND is performed to remove occult nodal metastases with negative preoperative assessments. Several studies have reported that patients who underwent prophylactic CND had a higher rate of complications and no effect on survival, and prophylactic CND may not be routinely recommended [9-12]. Furthermore, according to the 2015 American Thyroid Association (ATA) guidelines, prophylactic CND should be considered for patients with advanced primary tumors (T3 or T4) and clinically involved lateral neck nodes (cN1b), while CND may not be appropriate for small (T1 or T2), clinically node-negative (cNO) PTC [13]. In contrast, some studies have reported that prophylactic CND should be performed because of the high incidence of central neck LNM and the poor sensitivity of ultrasound [14]. In another study, of the patients who underwent prophylactic CND with cN0 PTC, $45.8 \%$ had central neck LNM, $0.9 \%$ had recurrent laryngeal nerve injuries, $1.16 \%$ had a locoregional recurrence, $6.24 \%$ had transient hypoparathyroidism, and $0.13 \%$ had permanent hypoparathyroidism [15]. Nevertheless, prophylactic CND in cNO PTC remains controversial.

Total thyroidectomy without CND helps to reduce the risk of hypoparathyroidism and recurrent laryngeal nerve injury, but it can leave metastatic LNs because of the high incidence of ipsilateral central compartment LNM. Finally, it can affect locoregional recurrence. In contrast, total thyroidectomy with bilateral CND has the advantage of identifying and controlling LNM, but it also has the disadvantage of increased risks of hypoparathyroidism and recurrent laryngeal nerve injury. The incidence of contralater- al LNM is below 5\%. If the risk factors for contralateral LNM are not present, ipsilateral CND may be reasonable. We focused on the extent of prophylactic CND during total thyroidectomies in cNO PTC patients. The endpoints of this study were (1) the incidence of transient and permanent hypoparathyroidism, (2) transient and permanent recurrent laryngeal nerve injury, and (3) recurrence. The aim of this study was to evaluate the benefits of prophylactic ipsilateral CND compared with bilateral CND in total thyroidectomy for $\mathrm{cNO}$ unilateral PTC.

\section{Materials and methods}

\section{Ethics statements}

This study was approved by the Institutional Review Board of Yeungnam University Hospital (IRB No: 2019-09-066).

\section{Patients}

We retrospectively enrolled 324 consecutive patients who underwent total thyroidectomies with prophylactic $\mathrm{CND}$ for $\mathrm{cNO}$ unilateral PTC by a single experienced surgeon between January 2009 and May 2010. The inclusion criteria were patients (1) with a final postoperative diagnosis of PTC, (2) who underwent a total thyroidectomy with prophylactic CND, (3) had follow-up parathyroid hormone (PTH) and calcium testing, and (4) had no evidence of clinical node positivity. The exclusion criteria were patients (1) without follow-up examinations or laboratory tests, (2) concomitant modified radical neck dissection, (3) parathyroidectomy due to parathyroid adenoma, (4) bilateral PTC, (5) 11 male sex in only group 1, and (6) other types of pathological results. One hundred-fifty patients (bilateral carcinoma, male, modified radical neck dissection, lack of follow-up or laboratory tests and parathyroidectomy) who met the exclusion criteria were excluded among of 324 patients. Finally, 174 patients were divided into two groups according to the extent of CND. Seventy-four patients in group 1 underwent prophylactic ipsilateral CND with total thyroidectomy and 100 patients in group 2 received prophylactic bilateral CNDs with total thyroidectomy. Ipsilateral CND was defined as the removal of ipsilateral paratracheal, pretracheal, and prelaryngeal LNs. Bilateral CND was defined as the removal of paratracheal, pretracheal, and prelaryngeal LNs. All patients received prophylactic ipsilateral CNDs and whether or not a prophylactic bilateral CND was performed depended on the frozen biopsy result of the ipsilateral $\mathrm{LN}$ and enlarged contralateral $\mathrm{LN}$ intraoperatively.

\section{Study design}

Patients with intraoperative findings, such as parathyroid color 
change and autotransplantation, were excluded because of subjective evidence. Robotic or endoscopic thyroidectomies were also excluded because those techniques offer magnified views that can make it easier for the surgeon to preserve the parathyroid. We classified PTC according to the American Joint Committee on Cancer 7th edition and minimal extrathyroidal extension was categorized as T3. The indications for radioactive iodine ablation were extrathyroidal extensions and LN involvement. We reviewed basic characteristics of the patients (age, tumor location, size, multifocality, concomitant benign tumor, and length of the lobe), histopathological factors (lymphocytic thyroiditis, extrathyroidal extension, margin status, number of retrieved LN, metastasis, and parathyroid on tissue) and laboratory tests (postoperative PTH and calcium). We investigated the incidence of transient and permanent hypoparathyroidism, transient and permanent hoarseness, and recurrence by reviewing the patients' medical records. Recurrence was defined as tumor in the thyroidectomy bed or metastatic central or lateral cervical LN after surgery. Recurrent laryngeal nerve injury was assessed by patient's self-assessment and physician's objective assessment. Permanent recurrent laryngeal nerve injury was defined as persistent hoarseness and vocal cord palsy identified with laryngeal examination more than 6 months after surgery. Through this study, we sought to find evidence for two hypotheses. The first hypothesis is that prophylactic CND during total thyroidectomy in cNO PTC should not be routinely discounted because of the rate of incidentally found CLNM. And second, prophylactic ipsilateral CND is more advantageous than bilateral CND according to the complication rate.

\section{Hypoparathyroidism}

Hypoparathyroidism was defined as PTH levels $<10 \mathrm{pg} / \mathrm{mL}$ (range, 10-65 pg/mL). Transient hypoparathyroidism was defined when a low PTH level returned to normal within the follow-up period. And permanent hypoparathyroidism was defined as PTH $<10 \mathrm{pg} / \mathrm{mL}$ with persistent low PTH levels during follow-up. PTH was tested on postoperative 1 day and then at annual follow-ups. Calcium levels less than $8.5 \mathrm{mg} / \mathrm{dL}$ were classified as hypocalcemia. We tested calcium level perioperatively, then regularly at follow-up. Calcium and vitamin D supplements were used to maintain the calcium and vitamin $\mathrm{D}$ levels as close to normal as possible, regardless of the patient's symptoms.

\section{Statistical analyses}

Chi-squared tests or Fisher exact test or linear and linear association was used to compare the categorical variables. Student t-tests were used for continuous valuables, which are expressed as mean \pm standard deviation. All statistical analyses were performed using IBM SPSS version 21.0 (IBM Corp., Armonk, NY, USA) and $p$-values $<0.05$ indicated statistical significance.

\section{Results}

\section{Patient characteristics}

Table 1 shows a comparison of the characteristics of patients between the two groups. One hundred seventy-four patients were included in this study, of which 74 were assigned to group 1 and 100 to group 2 according to the extent of CND. All 174 patients were female. The mean age of the patients was $47.8 \pm 11.2$ years and $45.1 \pm 10.2$ years and the mean tumor size was $0.8 \pm 0.5 \mathrm{~cm}$ and $0.9 \pm 0.6 \mathrm{~cm}$ in groups 1 and 2 , respectively. There was no significant difference in age, tumor size, location, multifocality, length of dominant lobe, minimal extrathyroidal extension, lymphocytic thyroiditis, positive margin or stage between the groups. However, radioactive iodine ablation was different in 31 patients $(41.9 \%)$ and 62 patients (62\%) in groups 1 and 2, respectively $(p=0.009)$. The mean follow-up period was $118.2 \pm 4.6$ and $115.5 \pm 4.1$ months in groups 1 and 2 , respectively $(p<0.001)$.

Table 1. Patient characteristics

\begin{tabular}{|c|c|c|c|}
\hline Characteristic & $\begin{array}{l}\text { Group } 1 \\
(n=74)^{a)}\end{array}$ & $\begin{array}{c}\text { Group 2 } \\
(n=100)^{b)}\end{array}$ & $p$-value \\
\hline Female sex & 74 & 100 & NA \\
\hline Age (yr) & $47.8 \pm 11.2$ & $45.1 \pm 10.2$ & 0.097 \\
\hline Age $<45$ yr & $28(37.8)$ & $42(42)$ & 0.580 \\
\hline Tumor size $(\mathrm{cm})$ & $0.8 \pm 0.5$ & $0.9 \pm 0.6$ & 0.736 \\
\hline Tumor location & & & 0.850 \\
\hline Upper & $14(18.9)$ & $24(24)$ & \\
\hline Middle & $52(70.3)$ & $65(65)$ & \\
\hline Lower & $5(6.8)$ & $6(6)$ & \\
\hline Isthmus & $3(4)$ & $5(5)$ & \\
\hline Multifocality & $14(18.9)$ & $16(16)$ & 0.614 \\
\hline Length of dominant lobe & $4.8 \pm 0.8$ & $4.6 \pm 0.7$ & 0.096 \\
\hline Minimal extrathyroidal extension & $43(58.1)$ & $66(66)$ & 0.287 \\
\hline Lymphocytic thyroiditis & $17(23)$ & $34(34)$ & 0.114 \\
\hline Margin positivity & $10(13.5)$ & $13(13)$ & 0.675 \\
\hline Stage & & & 0.614 \\
\hline I & $45(60.8)$ & $57(57)$ & \\
\hline III & $29(39.2)$ & $43(43)$ & \\
\hline Calcium replacement & $59(79.7)$ & $88(88)$ & 0.136 \\
\hline RI ablation & 31 (41.9) & $62(62)$ & 0.009 \\
\hline Follow-up (mo) & $118.2 \pm 4.6$ & $115.5 \pm 4.1$ & $<0.001$ \\
\hline
\end{tabular}

Values are presented as mean \pm standard deviation or number (\%). $N A$, not applicable; RI, radioactive iodine; CND, central neck dissection.

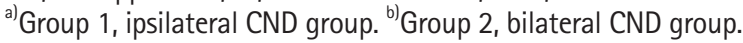




\section{Central neck dissection: ipsilateral versus bilateral}

The number of ipsilateral paratracheal LNs was $3.8 \pm 2.8$ and $4.8 \pm 3.1$ in groups 1 and 2 , respectively $(p=0.034)$. The number of pretracheal and prelaryngeal LNs were $0.6 \pm 1.2$ and $1.8 \pm 2.3$, respectively $(p<0.001)$. Ipsilateral paratracheal LNM was found in 21 patients (28.4\%) and 44 patients (44\%) in groups 1 and 2, respectively $(p=0.035)$. Pretracheal LNM was found in one patient $(1.4 \%)$ and 15 patients $(15 \%)$ in each group $(p=0.002)$. Contralateral paratracheal LNM was $10(10 \%)$ in group 2 . The final pathologic results showed that the parathyroid gland was inadvertently removed in 15 patients (20.3\%) and 22 patients (22\%) in groups 1 and 2, respectively. Among these, the ipsilateral parathyroid was removed in 12 patients $(16.2 \%)$ and 12 patients $(12 \%)(p=0.425)$ in groups 1 and 2 , respectively, the contralateral parathyroid in $2(2.7 \%)$ and $8(8 \%)(p=0.193)$ and both in 1 $(1.4 \%)$ and $2(2 \%)$. There was no significant difference in the removal of parathyroid glands inadvertently between the groups (Table 2).

\section{Postoperative parathyroid hormone and calcium levels}

The postoperative PTH and calcium level are shown in Table 3. Postoperative PTH levels were measured at 1 day, 1 year, and 2 years then annually at follow-up. By linear and linear association, there was a decreasing trend of the hypoparathyroidism $(\mathrm{PTH}<10 \mathrm{pg} / \mathrm{mL})$ over time $(p<0.001)$. The 1 -day PTH levels (average) were significantly different $(19.3 \pm 12.1$ in group 1 and $13.2 \pm 9.4$ in group $2 ; p<0.001$ ). One-year PTH levels (average) were $22.9 \pm 10.2$ and $19.6 \pm 10.6(p=0.003)$ and 2-year PTH lev-

Table 2. Comparison of ipsilateral versus bilateral prophylactic central neck dissection

\begin{tabular}{|c|c|c|c|}
\hline Variable & $\begin{array}{l}\text { Group } 1 \\
(n=74)^{a)}\end{array}$ & $\begin{array}{c}\text { Group 2 } \\
\left.(n=100)^{b}\right)\end{array}$ & $p$-value \\
\hline \multicolumn{4}{|l|}{ Numbers of retrieved LN } \\
\hline Ipsilateral paratracheal LN & $3.8 \pm 2.8$ & $4.8 \pm 3.1$ & 0.034 \\
\hline Pretracheal and prelaryngeal LN & $0.6 \pm 1.2$ & $1.8 \pm 2.3$ & $<0.001$ \\
\hline Contralateral paratracheal LN & 0 & $4.0 \pm 3.0$ & NA \\
\hline \multicolumn{4}{|l|}{ Metastasis in level VI LN } \\
\hline Ipsilateral paratracheal LN & $21(28.4)$ & $44(44)$ & 0.035 \\
\hline Pretracheal and prelaryngeal LN & $1(1.4)$ & $15(15)$ & 0.002 \\
\hline Contralateral paratracheal LN & 0 & $10(10)$ & NA \\
\hline \multicolumn{4}{|l|}{ Parathyroid in tissue } \\
\hline Ipsilateral & $12(16.2)$ & $12(12)$ & 0.425 \\
\hline Contralateral & $2(2.7)$ & $8(8)$ & 0.193 \\
\hline Both & $1(1.4)$ & $2(2)$ & NA \\
\hline
\end{tabular}

Values are presented as mean \pm standard deviation or number (\%). LN, lymph node; NA, not applicable; CND, central neck dissection.

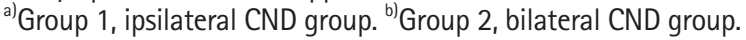

els (average) were 26.1 \pm 10.9 and $19.6 \pm 10.6$ in groups 1 and 2, respectively $(p<0.001)$. The calcium levels in groups 1 and 2 , respectively, were $9.1 \pm 0.5$ and $8.9 \pm 0.4$ preoperatively and $8.1 \pm 0.6$ and $7.8 \pm 0.6$ postoperatively. The perioperative calcium levels were not different $(1.0 \pm 0.7$ in group 1 and $1.1 \pm 0.6$ in group 2; $p=0.302$ ). But there was a significant difference in the incidence of postoperative severe hypocalcemia defined as calcium levels $\leq 7.0 \mathrm{mg} / \mathrm{dL}$ (6 patients [8.1\%] in group 1 and 23 [23\%] in group $2 ; p=0.009$ ).

\section{Hypoparathyroidism, recurrent laryngeal nerve injury, and recurrence}

Postoperative transient hypoparathyroidism was observed in 25 patients (33.8\%) and 38 patients (38\%) $(p=0.527)$, whereas permanent hypoparathyroidism occurred in three patients (4.1\%) and 19 patients (19\%) in groups 1 and 2, respectively. There was significant difference in the incidence of postoperative permanent hypoparathyroidism $(p=0.005)$. Transiently recurrent laryngeal nerve injury after total thyroidectomy with CND was estimated through medical record review, at 14 patients (18.9\%) and $17 \mathrm{pa-}$ tients $(17 \%)$ in groups 1 and 2 , respectively $(p=0.744)$. Permanently recurrent laryngeal nerve injury totally occurred in three patients (1.7\%). One patient in group 1 underwent nerve shaving because of recurrent laryngeal nerve invasion. In two patients in group 2, one patient received nerve repair and returned to a normal voice after 6 months. Laboratory tests and ultrasound examination were performed every 6 months until 2 years after surgery

Table 3. Postoperative PTH and calcium level

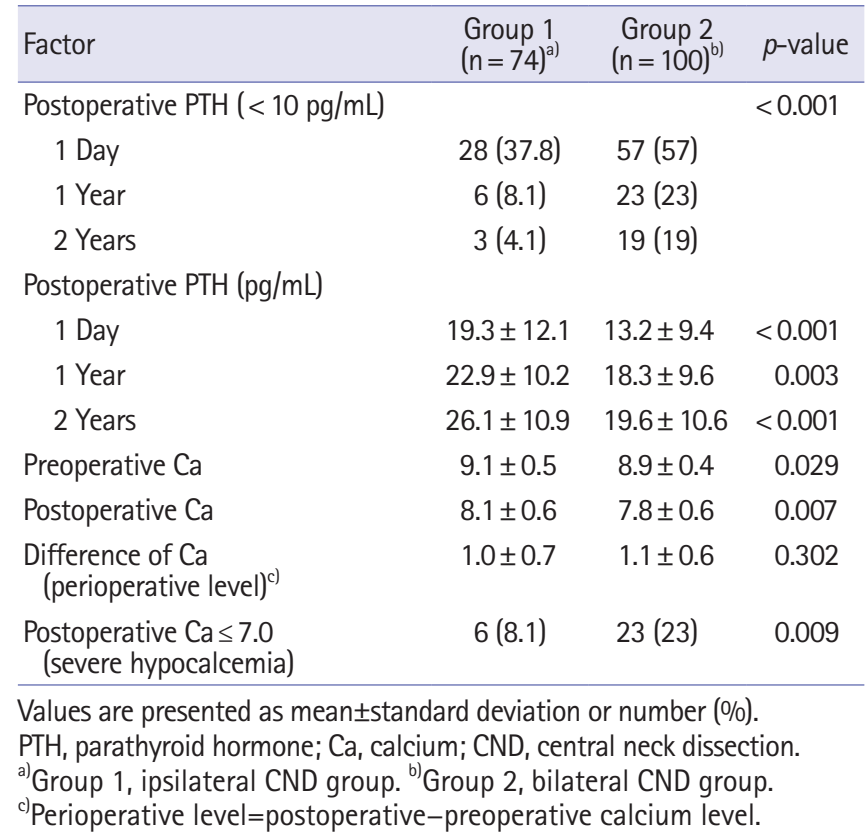


Table 4. Hypoparathyroidism, recurrent laryngeal nerve injury, and recurrence after total thyroidectomy with ipsilateral or bilateral CND

\begin{tabular}{lccc}
\hline Variable & $\begin{array}{c}\text { Group 1 } \\
\left.(n=74)^{\mathrm{a}}\right)\end{array}$ & $\begin{array}{c}\text { Group 2 } \\
(\mathrm{n}=100)^{\mathrm{b})}\end{array}$ & $p$-value \\
\hline $\begin{array}{l}\text { Hypoparathyroidism } \\
\quad \text { Transient }\end{array}$ & $25(33.8)$ & $38(38)$ & 0.527 \\
$\quad \begin{array}{l}\text { Permanent } \\
\text { Recurrent laryngeal nerve injury }\end{array}$ & $3(4.1)$ & $19(19)$ & 0.005 \\
$\quad$ & & & \\
$\quad \begin{array}{l}\text { Transient } \\
\quad \text { Permanent }\end{array}$ & $14(18.9)$ & $17(17)$ & 0.744 \\
Recurrence & $1(1.4)$ & $2(2)$ & NA \\
\hline
\end{tabular}

Values are presented as number (\%).

CND, central neck dissection; NA, not applicable.

${ }^{a}$ Group 1, ipsilateral CND group. ${ }^{b}$ Group 2, bilateral CND group.

and then annually during follow-up. Among the 174 patients, only two patients $(1.1 \%)$ had a recurrence. Contralateral paratracheal LNM was found in one patient in group 1, 8 years after surgery and another in group 2 was identified with lateral neck LNM 3.5 years after surgery (Table 4$)$.

\section{Discussion}

Prophylactic CND (ipsilateral or bilateral) can be considered in cN0 PTC with advanced tumors (T3 or T4) or cN1b [13]. Other study reported routine prophylactic $\mathrm{CND}$ is a treatment option for cNO PTC [15]. It remains controversial. Our study uniquely compared ipsilateral with bilateral CND according to the extent of prophylactic CND. Furthermore, no previous studies have reported the details of postoperative hypoparathyroidism among CND groups. The starting point of our study was whether the need for prophylactic CND could be discounted. Our study was subject to two well-known facts. First, there is a relatively higher possibility of complications in CND patients than in non-CND patients. Second, there is a high incidence of CLNM incidentally in cNO PTC patients. Surgeons might feel comfortable when only thyroidectomy is performed through capsular dissection, which can lead to decreased operative times and easy preservation of the parathyroid. Until now, the main idea was not to perform a prophylactic CND, which is prudent, except in the case of large tumors and clinically suspicious factors. Nevertheless, many endocrine surgeons might feel uneasy because of the high incidence of CLNM. If prophylactic CND is not preformed, the status and number of metastatic LNs cannot be determined. Ipsilateral CLNM might lead to contralateral central LN and lateral neck LNMs. Whether microscopic metastasis of the central LNs should be ignored is still debatable. However, it is clear that prophylactic CND has an important role in controlling metastatic LNs found incidentally. The incidence of recurrent laryngeal nerve injury can decrease according to the surgeon's experience but the preservation of the parathyroid is more complex. To preserve the parathyroid more experience and strong preservation intent are needed. CND is known to be associated with a higher incidence of hypoparathyroidism. We considered how to decrease the incidence of hypoparathyroidism and to get similar oncologic safety. Finally, we focused on prophylactic ipsilateral CND after considering the pros and cons of prophylactic CNDs. The aim of our study was to evaluate the benefits of prophylactic ipsilateral CND with total thyroidectomy in cNO PTC patients.

Several studies have reported the risk factors of central and lateral neck LNM $[7,14,16,17]$. Male sex, age ( $>45$ years), tumor size ( $>5 \mathrm{~mm}$ ), lower lobe, total tumor diameter $(>10 \mathrm{~mm})$, and extracapsular spread were statistically significant factors for CLNM in multivariate analysis [7]. One study showed that young age $(<45$ years), male sex, extrathyroidal extension, multifocal tumors, bilaterality, and tumor size $(\geq 1 \mathrm{~cm})$ were significant factors for CLNM, except in Hashimoto's thyroiditis [15]. Based on the above studies, the common risk factors for CLNM were young age, male sex, tumor size, and extrathyroidal extension. Furthermore, several studies found that CLNM was a significant risk factor for lateral neck LNM [18-20]. If some risk factors for CLNM are present preoperatively, prophylactic CND must be considered to control CLNM and reduce the possibility of lateral neck LNM. However, the role of prophylactic CND remains controversial, despite the high incidence of CLNM. A previous study found no significant difference in locoregional recurrence with or without $\mathrm{CND}$ and routine $\mathrm{CND}$ did not reduce lateral neck recurrence in $\mathrm{cNO}$ PTC patients [21]. Higher complication rates and similar recurrence rates were found in patients who underwent total thyroidectomies with CND than total thyroidectomies alone. Specifically, transient hypocalcemia was significantly higher in total thyroidectomies with CND [12]. CND is associated with higher rates of transient hypoparathyroidism (12.6\% in total thyroidectomy, $23.3 \%$ in total thyroidectomy with ipsilateral CND, and $36.7 \%$ in total thyroidectomy with bilateral CND) and a total thyroidectomy alone may be adequate treatment for patients with cNO PTC [21]. However, recently, one study reported that CND reduced locoregional recurrence (6.9\% difference at 10 years) [22]. Total thyroidectomy with CND resulted in a greater reduction in local recurrence than total thyroidectomy alone. Additionally, total thyroidectomy with bilateral CND showed significantly lower recurrence than total thyroidectomy alone [23]. This raises a question regarding how recurrence was assessed. Most studies used ultraso- 
nography to assess preoperative and recurrent CLNM. However, in our experience, most paratracheal LNs were classified as indeterminate by preoperative ultrasonography. Furthermore, we performed regular ultrasonographic examinations during follow-up. At that time, it was difficult to identify LNM by ultrasonography. Hypoechoic lesions or indeterminate LNs at paratracheal lesions were often found postoperatively which were difficult to completely assess, unless fine needle aspiration was performed. Actual CLNM recurrence might not be detected because of ambiguous ultrasonographic results. This hypothesis can explain why the noCND group had a recurrence rate similar to the CND group but a high incidence of CLNM. Preoperative ultrasonography has a 77\% detection rate for LNM. Detection of CLNM using ultrasound, which has $92 \%$ specificity, $81 \%$ positive predictive value, $51 \%$ sensitivity, and $76 \%$ negative predictive value, is difficult $[24,25]$. Another study also reported the poor diagnostic accuracy of ultrasound for CLNM. Prophylactic CND is recommended due to the low diagnostic efficacy of ultrasound and high incidence of CLNM [14]. As discussed above, prophylactic CND can remove up to $50 \%$ of the metastatic LN unidentifiable in ambiguous ultrasound results.

Hypoparathyroidism is a common complication after total thyroidectomy, which was transient in $6.9 \%-46 \%$ of the patients and permanent in $0 \%-10 \%$ of the patients. During surgery, the parathyroid gland can be damaged by thermal or mechanical injury, vascular injury, or removed inadvertently [26]. Permanent hypoparathyroidism is usually defined as PTH levels below normal for more than 1 year. However, in our study, it was defined as persistent PTH levels below normal at follow-up because there have been many cases where PTH levels were shown to improve or decrease after 1 year. Large tumors $(\geq 4 \mathrm{~cm})$ or gross extrathyroidal extension were significant factors for permanent hypoparathyroidism postoperatively. Of 65 patients who underwent total thyroidectomies with bilateral CND, postoperative transient and permanent hypoparathyroidism were found in $44(68 \%)$ and 12 (18\%), respectively [27]. Other study showed the risk factors of hypoparathyroidism following total thyroidectomy with CND. Of 903 patients who underwent total thyroidectomy plus CND, 399 patients $(44.2 \%)$ had transient hypoparathyroidism and 10 patients (1.1\%) had permanent hypoparathyroidism. On multivariate analysis, female, nonuse of carbon nanoparticles, parathyroid autotransplantation, accidental parathyroid resection and bilateral CND were the independent factors of transient hypoparathyroidism. Nonuse of carbon nanoparticles and a tumor in the upper pole of thyroid gland were significant risk factors for permanent hypoparathyroidism [28]. Our study found severe postoperative hypocalcemia (calcium level $\leq 7.0)$ in six patients $(8.1 \%)$ and 23 patients (23\%) in groups 1 and 2 , respectively $(p=0.009)$. Parathyroid was found on the tissue in 15 (20.3\%) of the ipsilateral CND patients and 22 (22\%) bilateral CND patients. Permanent hypoparathyroidism was found in three patients $(4.1 \%)$ and 19 patients $(19 \%)$ in groups 1 and 2 , respectively $(p=0.005)$. CND was associated with a higher incidence rate of hypoparathyroidism and complications. As shown in our study, 21 and $44 \%$ of the ipsilateral CLNM was found in CNO PTC patients incidentally. In contrast, the incidence of permanent hypoparathyroidism and severe hypocalcemia (calcium level $\leq 7.0$ ) in the ipsilateral CND group was significantly lower than in the bilateral CND group. The above results show that ipsilateral CND has the advantages of decreasing complication. A limitation of our study was that it was based on treatments performed 10 years ago. At that time, we focused on complete resection of the tumor burden, rather than preservation of the parathyroid. In conclusion, there were many cases of parathyroid present on removed tissue. Furthermore, we were notified of the loss of parathyroid inadvertently after surgery. If the inadvertent removal of parathyroid could be corrected, we could confidently state the advantages of ipsilateral CND. In group 2, there were higher incidence of RI ablation ( $41.9 \%$ vs. $62 \% ; p=0.009)$ and ipsilateral LNM (28.4\% vs. $44 \%$; $p=0.035$ ). These could be caused by advanced disease than extent of CND. There were many cases of total thyroidectomy despite tumor size less than $1 \mathrm{~cm}$, because of contralateral nodule or patient's preference. It might be a variable in determining the role of prophylactic ipsilateral CND.

In conclusion, our study showed lower permanent hypoparathyroidism and severe hypocalcemia rates in prophylactic ipsilateral CND compared with bilateral CND patients. Furthermore, there was no significant difference in recurrence rate between groups. We suggest that prophylactic ipsilateral CND may be safe and effective for selected patients undergoing a total thyroidectomy for cNO unilateral PTC.

\section{Acknowledgments}

\section{Conflicts of interest}

No potential conflict of interest relevant to this article was reported.

\section{Author contributions}

Conceptualization: all authors; Data curation: JGK, YAK; Methodology, Project administration: JGK; Writing-original draft: JGK; Writing-review \& editing: JEC, SJL. 


\section{ORCID}

Jin Gu Kang, https://orcid.org/0000-0002-3154-0697

Young Ah Kim, https://orcid.org/0000-0002-5289-3889

Jung Eun Choi, https://orcid.org/0000-0003-2290-6228

Soo Jung Lee, https://orcid.org/0000-0003-1202-3974

Su Hwan Kang, https://orcid.org/0000-0002-6508-006X

\section{References}

1. Sciuto R, Romano L, Rea S, Marandino F, Sperduti I, Maini CL. Natural history and clinical outcome of differentiated thyroid carcinoma: a retrospective analysis of 1503 patients treated at a single institution. Ann Oncol 2009;20:1728-35.

2. Rosato L, Avenia N, Bernante P, De Palma M, Gulino G, Nasi PG, et al. Complications of thyroid surgery: analysis of a multicentric study on 14,934 patients operated on in Italy over 5 years. World J Surg 2004;28:271-6.

3. Roh JL, Kim JM, Park CI. Central lymph node metastasis of unilateral papillary thyroid carcinoma: patterns and factors predictive of nodal metastasis, morbidity, and recurrence. Ann Surg Oncol 2011;18:2245-50.

4. Mulla M, Schulte KM. Central cervical lymph node metastases in papillary thyroid cancer: a systematic review of imaging-guided and prophylactic removal of the central compartment. Clin Endocrinol (Oxf) 2012;76:131-6.

5. Yan B, Hou Y, Chen D, He J, Jiang Y. Risk factors for contralateral central lymph node metastasis in unilateral cN0 papillary thyroid carcinoma: a meta-analysis. Int J Surg 2018;59:90-8.

6. Jiang LH, Chen C, Tan Z, Lu XX, Hu SS, Wang QL, et al. Clinical characteristics related to central lymph node metastasis in cN0 papillary thyroid carcinoma: a retrospective study of 916 patients. Int J Endocrinol 2014;2014:385787.

7. Xiang Y, Lin K, Dong S, Qiao LI, He Q, Zhang X. Prediction of central lymph node metastasis in 392 patients with cervical lymph node-negative papillary thyroid carcinoma in Eastern China. Oncol Lett 2015; 10:2559-64.

8. Zhang Q, Wang Z, Meng X, Duh QY, Chen G. Predictors for central lymph node metastases in CN0 papillary thyroid microcarcinoma (mPTC): a retrospective analysis of 1304 cases. Asian J Surg 2019;42:571-6.

9. Xu S, Liu W, Zhang Z, Liu Y, Xu Z, Liu J. Routine prophylactic central neck dissection may not obviously reduce lateral neck recurrence for papillary thyroid microcarcinoma. ORL J Otorhinolaryngol Relat Spec 2019;81:73-81.

10. Garcia A, Palmer BJ, Parks NA, Liu TH. Routine prophylactic central neck dissection for low-risk papillary thyroid cancer is not cost-effective. Clin Endocrinol (Oxf) 2014;81:754-61.
11. Sosa JA. Is routine prophylactic central neck dissection indicated for low-risk papillary thyroid cancer: can we determine cost-effectiveness if we are unsure about its effectiveness and safety? Surgery 2013;154:1146-7.

12. Lee DY, Oh KH, Cho JG, Kwon SY, Woo JS, Baek SK, et al. The benefits and risks of prophylactic central neck dissection for papillary thyroid carcinoma: prospective cohort study. Int J Endocrinol 2015;2015:571480.

13. Haugen BR, Alexander EK, Bible KC, Doherty GM, Mandel SJ, Nikiforov YE, et al. 2015 American Thyroid Association Management Guidelines for adult patients with thyroid nodules and differentiated thyroid cancer: the American Thyroid Association Guidelines Task Force on thyroid nodules and differentiated thyroid cancer. Thyroid 2016;26:1-133.

14. Zheng X, Peng C, Gao M, Zhi J, Hou X, Zhao J, et al. Risk factors for cervical lymph node metastasis in papillary thyroid microcarcinoma: a study of 1,587 patients. Cancer Biol Med 2019; $16: 121-30$.

15. Xue S, Wang P, Liu J, Li R, Zhang L, Chen G. Prophylactic central lymph node dissection in $\mathrm{cN0}$ patients with papillary thyroid carcinoma: a retrospective study in China. Asian J Surg 2016;39:131-6.

16. Mao LN, Wang P, Li ZY, Wang Y, Song ZY. Risk factor analysis for central nodal metastasis in papillary thyroid carcinoma. Oncol Lett 2015;9:103-7.

17. Konturek A, Barczynski M, Nowak W, Wierzchowski W. Risk of lymph node metastases in multifocal papillary thyroid cancer associated with Hashimoto's thyroiditis. Langenbecks Arch Surg 2014;399:229-36.

18. Wu X, Li B, Zheng C, He X. Predicting factors of lateral neck lymph node metastases in patients with papillary thyroid microcarcinoma. Medicine (Baltimore) 2019;98:e16386.

19. Ryu YJ, Kang SJ, Cho JS, Yoon JH, Park MH. Identifying risk factors of lateral lymph node recurrence in clinically node-negative papillary thyroid cancer. Medicine (Baltimore) 2018;97: e13435.

20. Gong Y, Yang J, Yan S, Su A, Liu F, Gong R, et al. Pattern of and clinicopathologic risk factors for lateral lymph node metastases in papillary thyroid carcinoma patients with lateral cervical lymphadenopathy. Medicine (Baltimore) 2018;97:e12263.

21. Calo PG, Conzo G, Raffaelli M, Medas F, Gambardella C, De $\mathrm{Crea} \mathrm{C}$, et al. Total thyroidectomy alone versus ipsilateral versus bilateral prophylactic central neck dissection in clinically node-negative differentiated thyroid carcinoma. A retrospective multicenter study. Eur J Surg Oncol 2017;43:126-32.

22. Barczynski M, Konturek A, Stopa M, Nowak W. Prophylactic central neck dissection for papillary thyroid cancer. Br J Surg 
2013;100:410-8.

23. Liu H, Li Y, Mao Y. Local lymph node recurrence after central neck dissection in papillary thyroid cancers: a meta analysis. Eur Ann Otorhinolaryngol Head Neck Dis 2019;136:481-7.

24. Kim E, Park JS, Son KR, Kim JH, Jeon SJ, Na DG. Preoperative diagnosis of cervical metastatic lymph nodes in papillary thyroid carcinoma: comparison of ultrasound, computed tomography, and combined ultrasound with computed tomography. Thyroid 2008; 18:411-8.

25. Roh JL, Park JY, Kim JM, Song CJ. Use of preoperative ultrasonography as guidance for neck dissection in patients with papillary thyroid carcinoma. J Surg Oncol 2009;99:28-31.
26. Canu GL, Medas F, Longheu A, Boi F, Docimo G, Erdas E, et al. Correlation between iPTH levels on the first postoperative day after total thyroidectomy and permanent hypoparathyroidism: our experience. Open Med (Wars) 2019;14:437-42.

27. Teshima M, Otsuki N, Morita N, Furukawa T, Shinomiya H, Shinomiya $\mathrm{H}$, et al. Postoperative hypoparathyroidism after total thyroidectomy for thyroid cancer. Auris Nasus Larynx 2018; 45:1233-8.

28. Su A, Wang B, Gong Y, Gong R, Li Z, Zhu J. Risk factors of hypoparathyroidism following total thyroidectomy with central lymph node dissection. Medicine (Baltimore) 2017;96:e8162. 